\title{
Atomic Force Microscopy for Collagen-Based Nanobiomaterials
}

\author{
Andreas Stylianou \\ Cancer Biophysics Laboratory, Department of Mechanical and Manufacturing Engineering, Faculty of Engineering, \\ University of Cyprus, Nicosia 1678, Cyprus \\ Correspondence should be addressed to Andreas Stylianou; styliand@mail.ntua.gr
}

Received 1 August 2016; Revised 28 November 2016; Accepted 23 January 2017; Published 15 February 2017

Academic Editor: Ilaria Armentano

Copyright (C) 2017 Andreas Stylianou. This is an open access article distributed under the Creative Commons Attribution License, which permits unrestricted use, distribution, and reproduction in any medium, provided the original work is properly cited.

Novel nanobiomaterials are increasingly gaining ground in bioengineering research. Among the numerous biomaterials, collagennanobiomaterials, such as collagen thin films, are of great interest since they present a wide range of applications in the fields of biomaterials, tissue engineering, and biomedicine. Collagen type I is the most abundant protein within extracellular matrix and, due to its unique characteristics, is widely used as biomaterial. A thorough characterization of the structure and properties of nanomaterials can be achieved by Atomic Force Microscopy (AFM). AFM is a very powerful tool which can be used to obtain qualitative or quantitative information without destroying the collagen fibrillar structure. This mini review covers issues related to the use of AFM for studying the structure and mechanical properties of collagen-based nanobiomaterials, collagen-substrate interactions during the formation of collagen thin films, collagen-cells interactions, and the collagen-optical radiation interactions.

\section{Introduction}

The development of nanobiomaterials and nanotechnologybased methods is cutting edge research in many disciplines having multiple applications such as functional surfaces and biosensors, while they can be used as extracellular matrix models. Collagen-nanobiomaterials, such as collagen thin films, are of great interest since they have broad applications in the fields of biomaterials, tissue engineering, and biomedicine.

Collagens comprise almost $30 \%$ of total cell protein in mammalian cells and they have been identified among the most promising biomaterials for the formation of novel bioengineering interventions [1-3]. Although the superfamily of vertebrate collagen includes over 50 collagen and collagen like proteins $[4,5]$, collagen type I presents great interest due to its particular characteristics, such as ability for selfassembly, biocompatibility, biodegradability, and nontoxicity [6]. Collagen type I is the most abundant protein in mammals and is the major protein in the extracellular matrix [7] and its importance in vertebrate biology is crucial [8]. It is fibrous in nature, and its molecule consists of three amino acid chains that form rod-shaped triple helices assembled to form fibrils $[4,9]$. These fibrils are then aligned to form more complex structures, like bundles and fibers (see Figure 1(a))
$[4,7,9]$. Collagen molecules are packed in a quarterstaggered fashion which gives rise to a repeating banding pattern the so-called D-periodicity or D-band, of about $67 \mathrm{~nm}$ (see Figure 1(b)) $[1,5,10,11]$. The exact length of the Dband depends on the tissue $[1,5,12,13]$. It has been reported that the transverse $\mathrm{D}$-banding periodic pattern is key player concerning fibril mechanical properties, while it has been correlated with pathological conditions and it is believed to play a significant role in cell-collagen interactions [12-15]. For instance, a strong correlation between the orientation of $\mathrm{D}$ band and cell elongation has been reported [14]. Collagen type I fibrils are the elementary building blocks in many collagen-rich tissues $[16,17]$ and collagen has a wide range of functions, from tissue mechanical strength and scaffolding to cell migration and tissue repair $[1,4,18]$. Collagen presents different morphologies in different tissues [1]. For instance, in skin dermis, collagen is in the form of loosely interwoven, wavy, randomly oriented, and loosely packed bundles while in tendon, collagen has a uniform distribution of fibril diameters with a lateral packing [19]. Ubiquitous within the extracellular matrix, collagen acts to maintain the shape and integrity of tissues [1].

The control of collagen self-assembly process enables the formation of collagen-based biomaterials that can serve 


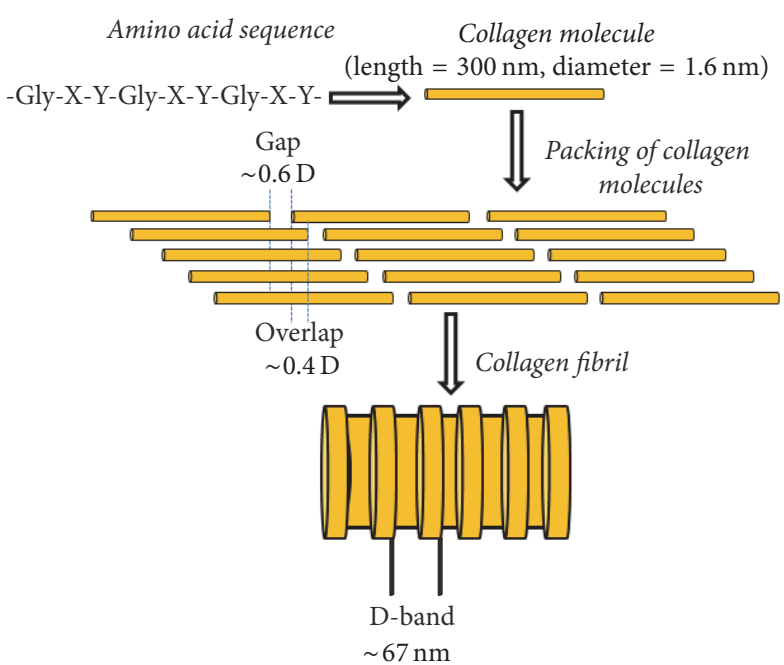

(a)

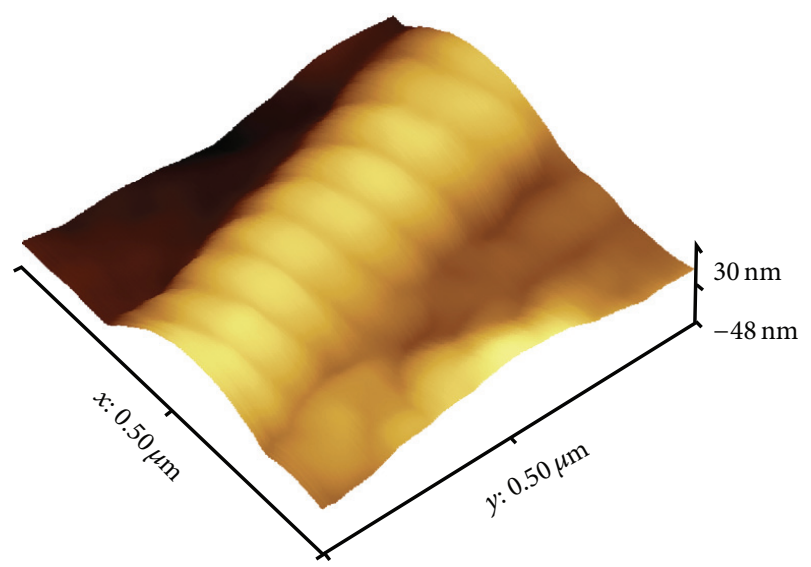

(b)

Figure 1: (a) The collagen structure from amino acid sequence to fibril. In this panel the amino acid sequence, the collagen molecule, the packing of collagen molecules, and the structure of a collagen fibril are presented. (b) A collagen fiber D-band periodicity from a collagen thin film imaged using Atomic Force Microscope (CPII Veeco-Bruker Microscope) in tapping mode.

as an in vitro model of different collagen-rich tissues. In order to enhance collagen biomaterials performance and their possible applications a thorough characterization of their structure and properties is required at a nanoscale level. Such a characterization can be performed by Atomic Force Microscopy (AFM) (see Figure 2) [20, 21]. AFM is a scanning probe microscope (SPM) that records interactions between a probe (the AFM tip) and the sample surface. Since its invention in 1980s, it has become a fundamental technique in the fields of surface and biomedical science. AFM is unique and has several advantages over the other microscopy techniques, such as Scanning or Transmission Electron Microscopy (SEM and TEM) and optical microscopy (including fluorescent and Confocal Laser Scanning Microscopy). First of all, AFM provides topological information at nanoscale level that other microscopes cannot measure. For instance, AFM can perform mechanical properties characterization of the samples at a nanoscale level and can offer a combination of qualitative and quantitative information. Furthermore, AFM possesses the ability to perform nanoscale imaging/characterization without the need for presence of vacuum conditions or any special treatment of the specimen, such as sample labeling with antibodies/fluorescent labels or surface coating $[8,22$, 23]. The AFM characterization can be performed without destroying the fibrillar structure of collagen. AFM can be used for investigating a wide range of collagen-based structures, from collagen molecules to separated fibrils and fibers and collagen-based nanobiomaterials as well $[6,24]$.

In this review, we first introduce the AFM apparatus and its working principle and we then focus on its application on the study of collagen thin films. This short review covers issues that are relevant to the use of AFM and collagen thin films for studying (a) collagen-substrate interactions during collagen thin film formation, (b) collagen-cells interactions, and (c) collagen-optical radiation interactions. Also, the use of AFM for studying three-dimensional (3D) collagen gels is briefly presented. As there is always a need to develop novel optical biomarkers and/or imaging modalities, it is imperative to better understand the collagen-optical properties and optical radiation interactions $[25,26]$. Although the general properties are well known $[27,28]$, the exact mechanism in many cases, like nonlinear optical properties of collagen (e.g., second harmonic generation-SHG), is not fully clarified $[29,30]$. Furthermore, the study of collagenoptical radiation interactions will enhance better collagen biomaterial performance as some optical radiation-collagen interactions, like ultraviolet (UV) irradiation of collagenbased materials, are used as sterilizing procedures [31, 32].

\section{Atomic Force Microscopy}

The AFM is the second member (after the scanning tunneling microscope-STM) of the SPM family [22]. AFM was developed in 1986 by Binnig and colleagues [33] and commercial AFMs began to appear in the early 1990s [22]. Since its invention it has rapidly become a popular method for nanoscale imaging and mechanical properties characterization of a broad range of samples, including biological ones [34, 35]. AFM is considered as a versatile and powerful microscope because it is the only microscope that not only offers highresolution imaging (even within atomic resolution), but also provides various types of surface measurements. AFM gathers the information with a probe, which is a very sharp tip that is mounted on a cantilever. The probe scans the sample surface and measures the atomic forces between probesample surfaces (see Figure 2). AFM apparatus traditionally consists of a laser beam deflection system (optical lever) where a laser beam is reflected from the back of the cantilever (on which a tip is mounted and come into contact with surface) and onto a position-sensitive detector (see Figure 2). 


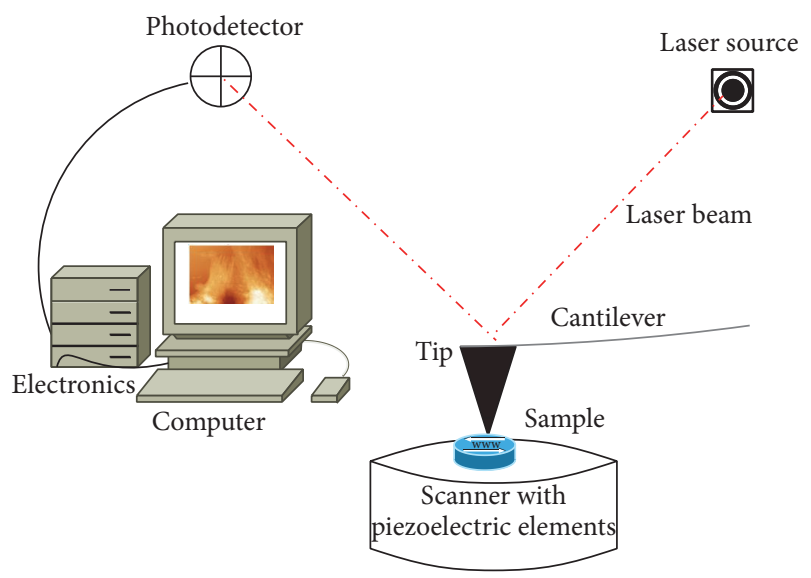

FIGURE 2: AFM apparatus. Generally an AFM consists of a probe (cantilever and tip), a laser source, a scanner, a photodetector, the electronics, and a computer.

The accurate movement of the tip over the surface or sample under the tip (depending on the system) is achieved with piezoelectric elements. Also, the movement is monitored by the alterations of the laser spot position on a photodetector. The measurements of the tip deflection are then transferred to the system's electronics and computer which use them to form three-dimensional (3D) images of the samples or to assess other sample's properties, such as stiffness and adhesion [33].

The fact that AFM demands minimum sample preparation (e.g., does not require dehydration, sample labeling with fluorescent dyes or antibodies, or surface coating), is a nondestructive technique, and can operate under different conditions (air and liquid) [23] makes it very powerful for biological and bioengineering studies [34-36]. Also, it operates in many different modes, offering a vast amount of qualitative and quantitative information about biosamples $[8,37,38]$ ranging from topography to mechanical properties characterization [20,39-41]. The most well-known AFM modes are the contact mode (where the tip is always in contact with the sample surface), the tapping mode (some manufactures call it intermittent or AC mode (where tip taps the surface and touches the sample surface only for a short time)), and the noncontact mode (where the tip does not come in contact with the sample surface). For biological samples (that are very soft), tapping mode is more frequently used as it is less destructive than contact mode and it is more easily performed than noncontact mode, while problematic issues with lateral and frictional forces are minimized [20, 34].

Finally, AFM can perform force spectroscopy for measuring mechanical properties of the samples. In AFM force spectroscopy, the cantilever-tip assembly can act as a force sensor [42] so as to provide several modes for measuring mechanical properties of the sample [43]. By using the AFM nanoindentation procedure [44-46] or the so-called force scanning mode [47] the hardness or softness of the specimen can be recorded and Young's modulus maps of the sample's surface can be generated [39, 41, 48-50]. More specifically, in the AFM nanoindentation method, indentation-force curves are formed. Then these curves are fitted using mathematical expressions (e.g., the Hertz model) so as to calculate values that are needed for measuring the sample's Young modulus and for forming material's properties maps (e.g., Young's modulus maps) [51, 52].

Although AFM is a powerful microscope, there are many technological challenges (e.g., instrumentation, experiment setup, and procedures) to be overcome for its better performance [53-58]. The majority of AFM procedures are time consuming and require intensive labor, which is a significant drawback since the achievement of statistical significant results is very challenging [55]. Additionally, further research is required for the improvement of the existing mathematical models that are used for the acquisition of quantitative data with AFM modes [59].

\section{AFM and Collagen-Based Nanobiomaterials}

Since cell adhesion and proliferation are affected by biomaterials nanocharacteristics and as almost all the biological reactions take place on surfaces or interfaces, the surface properties of biomaterials are a crucial parameter in biomedicine $[60,61]$. It has been demonstrated that dewetting, drying, and rehydrating collagen thin films do not influence their characteristics and consequently these films can be used as cell culturing substrates [62]. In the next section we summarized issues related to the use of AFM for studying collagen thin films formation, collagen-substrate interactions, collagen thin films, and cell interactions. Also the combination of collagen thin films and AFM for studying collagen-optical radiation interactions is presented.

3.1. Collagen Thin Films Formation. The methods that can be used for the formation of collagen thin films are of critical importance for the formation of the final collagen-based biomaterial. The formation of collagen-based biomaterials with predetermined and tunable natural characteristics is a very challenging task. Although the general principles of the collagen self-assembly are known, the exact mechanism of the process is not well defined [17]. Collagen self-assembly is an entropy-driven process which affects both the structure and the diameters of the fibrils [1]. This process is responsible for the unique D-periodicity pattern of collagen [1] and, in vivo, it takes place by the appropriate arrangement of thin fibrils in recesses that are located in the cell membrane. On the other hand, in vitro, the process occurs by both linear and lateral growth steps with parallel events that are seen in vivo [63]. Moreover, in vitro, the mechanism is significantly altered, since collagen self-assembly takes place without any cellular control or the presence of the enzymatic cleavage of the propeptides [63]. Thus, the formation of collagen-based biomaterials is not straightforward and here is where AFM methods can significantly contribute towards the evaluation of the final material and studying the collagen-substrate interactions as well.

A variety of different approaches, including drop casting from solution, the spin coating process, and the use of hydrodynamic flow, can be applied in order to form pure collagen two-dimensional (2D) thin films. A number of 
studies have demonstrated that each of these approaches allows the formation of films with different characteristics $[8,20,38]$. The method of drop casting from solution allows the formation of films that are relatively thick and homogeneous. Furthermore, these films consist of random oriented collagen fiber/fibrils and with collagen aggregations. By using the spin coating process ultrathin and homogeneous collagen films, with fibrils presenting the natural D-band (see Figure 1(b)) and with random orientation (see Figure 3(a)), can be formed. Finally, the hydrodynamic flow methodology leads to the formation of collagen thin films consisting of welloriented fibrils. This methodology has been used for very low collagen concentrations $(0.3 \mu \mathrm{g} / \mathrm{mL})$ and for collagen solutions consisting from more than one type of collagen (such as types I and III) or in the presence of sodium or potassium with very good results $[14,24,64,65]$. This formation methodology enables the formation of nanostructured thin films with tunable characteristics. Characteristics such as tubes, grooves, and fibers' orientations are appropriate for the study of cell behavior due to their interactions with substrates with different characteristics. Of course, there are many other more sophisticated and complex methodologies that can be used for the formation of oriented collagen thin films, such as nanoimprinting [66] and electrospinning [67], which are beyond the scope of this review.

3.2. Collagen-Substrate Interactions. Apart from the formation methodologies, the substrate that is used for the formation of 2D collagen thin films is a significant parameter for the nanocharacteristics of the collagen film. Among the different substrates that are found in the literature, mica discs or sheets are the most frequently used substrates that are used for studying the collagen-substrate interactions with AFM.

Traditionally, in AFM studies, mica surface is used as specimen substrate, due to its atomically flat and clean surface after cleavage [22]. In the case of the formation of collagen-based films mica characteristics can also affect the film formation [68, 69]. Mica consists of a series of thin crystalline plates that can easily be split apart ("cleaved") [22]. After cleavage, mica sheets are atomically flat and serve as a truly fresh surface that has not been exposed to the atmosphere and does not demand further removal of contamination $[22,70]$. The most frequently type of mica that is used as an AFM substrate is the "muscovite" mica, $\mathrm{KAl}_{2}(\mathrm{OH})_{2} \mathrm{Si}_{3} \mathrm{O}_{10}$. The muscovite mica is composed of negatively charged aluminosilicate layers in which the negative charge of the layers arises from a substitution of a quarter of the $\mathrm{Si}^{4+}$ ions by $\mathrm{Al}^{3+}$ ions. These layers are kept together by electrostatically bound potassium ions $(\mathrm{K}+)$ [71]. It has been demonstrated that upon cleavage the potassium layer is disrupted $[70,71]$ and the mica surface exhibits a hexagonal arrangement of $\mathrm{Si}$ and $\mathrm{O}$ atoms, while it can be partly covered by potassium ions [71]. Mica is negatively charged in aqueous liquids and due to the fact that it is negatively charged even at $\mathrm{pH} \sim 3$ [72] it serves as a hydrophilic surface model that can strongly affects the sample structure $[69,73]$. Furthermore, silanization protocols can be used in order to transform this well-used surface [71]. In the case of collagenmica interactions, there are many and strong interactions between these materials [74] since collagen is characterized by a complex charge distribution [75]. Additionally, it has been demonstrated that collagen self-assembly occurs easier when collagen assembles on a hydrophilic surface such as mica [76]. Also, it has been reported that the formation of oriented fibrils on mica substrates is a consequence of the hydrodynamic film deposition [64] and the crystallographic orientation of the mica substrate [74]. The crystalline nature and the surface properties of mica also appear to allow better adsorption of collagen fibers and the formation of fibrils with physiological characteristics, such as the D-periodicity of $\sim 67 \mathrm{~nm}$.

Moreover, of particular interest are the surfaces of polystyrene particles (polystyrene particle surfaces) that can be formed on mica and used as substrates for collagen thin film formation [20,37]. By using polystyrene nanoparticles on fresh cleaved mica and by optimizing the experimental parameters, the formation of uniform nanostructured surfaces can be achieved (see Figure 3(b)). These surfaces were shown to be suitable substrates for thin collagen film formation exhibiting physiological characteristics. Moreover, they can be used as extracellular matrix models for investigating the relationship between specific surface nanocharacteristics and the adsorption of collagen fibrils or other proteins.

Consequently, the use of different substrates and different thin films formation methodologies allows the development of collagen thin film with predetermined surface characteristics. The characterization, the quantification, and the control of the collagen thin films nanocharacteristics allow the films to be used as models for the investigation of the effect of the surface characteristics on cell behavior and the study of the effects of optical radiation on collagen.

3.3. Collagen Thin Films and Cell Interactions. Surfaces with well-organized geometry can be used for biomolecular interaction analysis and collagen-based surfaces that have fibers in alignment or porous structures have better performance and can influence or even drive cells behavior [77, 78]. Additionally, Plant et al. suggested that some of the mechanical cues that cells receive from their environment exist also in cells-thin films interactions and their thin film model achievement to elicit equivalent responses in cells as do bulk collagen gels [79]. The movement of cells towards specific directions and their orientation present a significant research interest since these characteristics are crucial in wound-healing and metastasis. The directional motion of cells along the axis of fibrous structures on aligned extracellular matrix (ECM) components (such as collagen) has been related to the so-called "contact guidance mechanism" [80]. Concerning collagen and collagen-based materials, the exact mechanism and the nature of the structural cue within the collagen fibers/fibrils that can drive cell movement are unknown. The D-band periodicity has been suggested to be a crucial player in the cell-collagen interactions since it seems that cells respond to information contained in this periodic pattern [14]. It is believed that the morphodynamics of cells are triggered by a combination of surface features and the cellular environment's mechanical properties [79]. Furthermore, Loesberg et al. demonstrated that there is a 


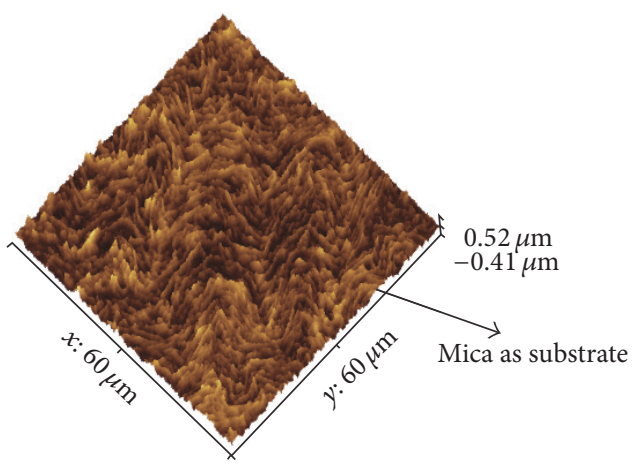

(a)

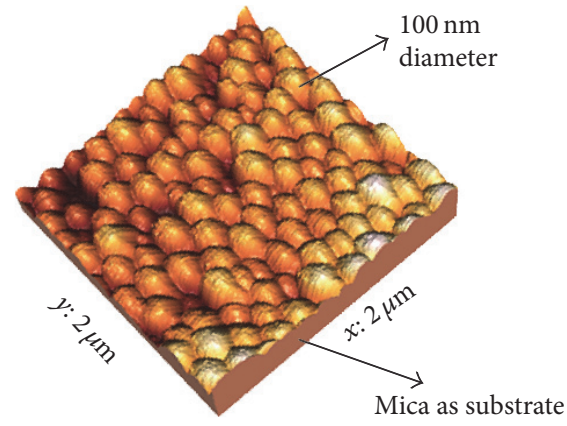

(b)

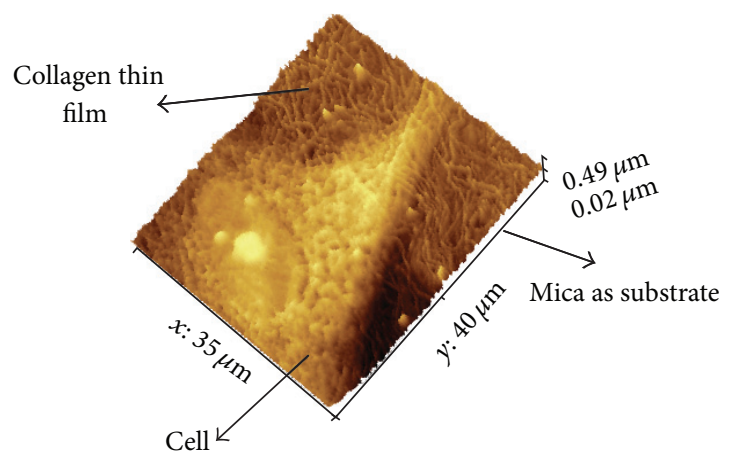

(c)

Figure 3: (a) A collagen thin film consisting of random oriented collagen fibers, (b) a polystyrene particle surface, and (c) a fibroblast lying on a collagen thin film.

threshold limiting the cells' response mechanism to contact showing that for fibroblasts cultured on a nanotopography substrate the threshold was found to be $35 \mathrm{~nm}$ [81]. In another work, collagen-based biological models were used as substrates for the growth of primary culture of human dermal fibroblasts [35] (see Figure 3(c)). The results showed that the films can be used as cell culture substrates and that fibroblasts respond to the topography of the films. When films with oriented collagen fibers were used, it was demonstrated that the fibroblasts followed the main orientation of the collagen fibers. Moreover, on films with randomly oriented fibers, the fibroblasts showed no standard arrangement in the available space during their growth. AFM enabled the simultaneous imaging of both fibroblasts and collagen fibers, so as to qualitatively present the relationship between the fibroblast orientation and the characteristics of the substrate [35]. In contrast, cells that were cultured on plain native mica did not grow normally and were characterized by a nonphysiological globular shape [14, 35, 82]. In addition, AFM multimode imaging (which is the imaging using more than one AFM mode, like the combination of topography with AFM phase imaging and mechanical properties characterization) has been used to investigate the heterogeneous structure and the mechanical properties of collagen fibers in thin films as well as that of fibroblasts cultured on collagen thin films [40, 49].

Consequently, AFM can significantly contribute towards the fully clarification of the existing mechanisms of cell-nanomaterials interactions so as to enable the design and development of novel nanobiomaterials with improved properties.

\subsection{Collagen Thin Films for Studying Collagen-Optical Radia- tion Interactions}

(i) Collagen Thin Films and Ultraviolet Irradiation. The investigation of collagen-ultraviolet (UV) radiation interactions is of crucial importance since the UV rays from sun interact with humans' collagen-based tissues on a daily basis, while UV light has been identified as a significant causative factor for skin aging and a number of skin cancers [83-85]. Moreover, UV is frequently used in order to sterilize or cross-link biomaterials [86-88]. Not surprisingly, UV light can modify a number of collagen characteristics, including its mechanical properties, structure, and chemical stability [89-93]. The use of AFM can significantly help in the research in this direction and especially on issues related to surface properties, which are very important in biomaterials.

It has been demonstrated that the characterization of collagen thin films with AFM allowed the exploration of the effects of UV irradiation $(254 \mathrm{~nm})$ on the optical properties of collagen (fluorescence, absorption), its topography, roughness, and the influence on cell culturing $[94,95]$. It must be noticed that surface roughness is a crucial factor in biomedical applications and plays a significant role in cellsurface crosstalk $[96,97]$ as alterations in roughness change the surface that is available for cell adhesion and growth [98]. It has been shown that for UV irradiation of the order of magnitude as those used for sterilization/crosslinking purposes, photodegradation occurs and AFM imaging demonstrates 
modifications in collagen surface roughness [95]. Also, it was demonstrated that fibrils retained their characteristic band and structure D-periodicity (see Figure 4). On the other hand, for long irradiation times and consequently for higher light doses, UV altered significantly both structure and surface roughness. Also, when UV irradiation was applied on collagen solution significantly different effects were recorded compared with those after films irradiation. It was suggested that the noticed changes in the fluorescence and absorption spectrum were due to the photodegradation caused by UV radiation. The photodegradation were correlated to structural changes in aromatic amino acids residues, while it is believed that UV caused the breaking of the peptides bonds and subsequently increased the formation of tyrosine and phenylalanine photoproducts. Furthermore, modifications in the surface roughness were recorded. These changes suggested that this technique can be used as a methodology for controlling the surface roughness during the formation procedure of collagen-based nanobiomaterials. The decrease of surface roughness of pure collagen films was also demonstrated by Sionkowska et al. [99]. Also, it has been demonstrated that for low UV doses (in the range of the sterilizing doses) surface roughness was decreased in films formed by collagen blends, like collagen-PVA (poly-vinyl alcohol) [99], collagen-poly-vinyl pyrrolidone (PVP) [100], and collagen-poly(ecaprolactone) (PCL) [101]. Additionally, AFM and contact angle studies have been applied in order to show the UV damage of collagen in polymer films containing a small amount of collagen [93].

In the case of the use of UV-irradiated collagen films as substrates for culturing cells, it has been shown that the cells' behavior is affected by the UV irradiation of collagen films $[95,102,103]$. It was demonstrated that for low irradiation doses (relevant to those used for sterilizing purposes) cells/fibroblasts can spread on irradiated thin films. Furthermore, when the irradiation time period was increased both the bodies and the nuclei of fibroblasts became increasingly globular, a fact which is a characteristic of fibroblasts' abnormal growth [95]. Previous studies with SEM have also shown this tendency of the cells to become more spherical when they were cultured on UV-irradiated collagen substrates [104]. Furthermore, in the literature, it was demonstrated that cell growth was supported by the UV irradiation of collagen gels, with negative results concerning cell proliferation $[102,103]$. In contrast, a noticeable enhancement in cell viability in UV-irradiated collagen blends has also been demonstrated [93]. This improvement in cell behavior has been proposed as a consequence of the UV-crosslinking that takes place, while it has also been associated with the possibility that some hidden recognition motifs of the cells were revealed $[103,105]$. Moreover, since for low irradiation doses the AFM studies revealed that there were not significant alterations in topography, the changes in cell behavior may be due to either mechanical properties alterations and/or chain scission $[106,107]$. In this direction, in a recent study, AFM was applied to record load-displacement curves on both the gap and overlap zones of collagen $\mathrm{D}$-spacing under the influence of UV irradiation [41].
The results demonstrated that the UV rays have a noticeable influence on the height level differences between the overlapping and gap zones. Additionally, it was shown that UV rays influenced the mechanical properties of collagen fibrils and the elastic values, in terms of Young's modulus, were reduced in D-spacing zoned. These results were correlated to the UV-induced polypeptide chain scission. Consequently, all the previous research results suggest that UV irradiation should be avoided or kept to the minimum possible levels in the case of collagen-based biomaterials [95].

(ii) Collagen and Low-Level Red Laser. Low-Level Laser Therapy (LLLT) can be used to treat a range of pathological conditions, including healing wounds. A recent study was focused on the effects that Low-Level Red Laser (LLRL) has directly on collagen and the influence on cell culturing [108]. The experimental results of LLRL $(661 \mathrm{~nm})$ on collagen thin films showed that although a slight increase in the emitted fluorescence intensity was measured, no alterations were observed in the nanotopography of collagen films after irradiation [108]. It should be noted that the parameters of irradiation (wavelength, dose, and irradiation time intervals) were of the order of magnitude as those used for the treatment with a low power laser. Although collagen topography was not altered, the results demonstrated that irradiation of LLRL had a negative effect on cell growth due to the effects of irradiation on collagen. By increasing the irradiation dose offered in collagen thin films, fibroblasts became more and more spherical. This is an evidence of abnormal growth of the fibroblasts, as it was also observed in the UV-irradiated films. Since the modulation of cellular behavior was observed in the absence of measurable surface changes, the alteration of cell behavior due to LLRL irradiation is not affected by the "surface guidance mechanism" (which relies on the surface properties of the substrate). This result represents important and novel information that offers new data towards the clarification of the LLLT mechanism.

(iii) Collagen Thin Films and Second Harmonic Generation. Collagen thin films can be used as ECM model for investigating the mechanism of the second harmonic generation (SHG), by near infrared (NIR) laser stimulation. SHG is a wellknown nonlinear optical process. During this phenomenon, photons with the same energy (same frequency $f$ ) interact with a noncentrosymmetric material (such as collagen) and as a result new photons with frequency equal to $2 f$ are generated [109]. In order to enhance this phenomenon, laser sources are used since the highly focused laser beams also provide high intensities and monochrome photons. SHG is emerging as a novel technique due to its noninvasiveness nature, the absence of external dyes, and the selectivity to specific tissue components [110-113]. Collagen is probably the most important component of tissues that can emit very bright SHG signals since it is a natural noncentrosymmetric biomaterial [30, 112, 114-117]. Consequently, novel SHGbased nonlinear methods that can be used for the diagnosis of a number of pathological conditions that are related to collagen fibers differentiation (such as alterations in collagen concentration, structure, and function) can be developed 


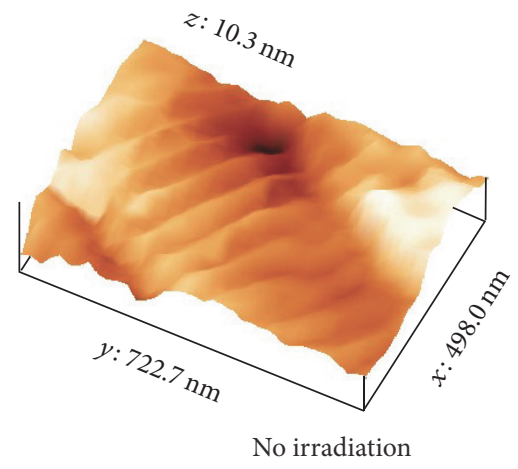

(a)

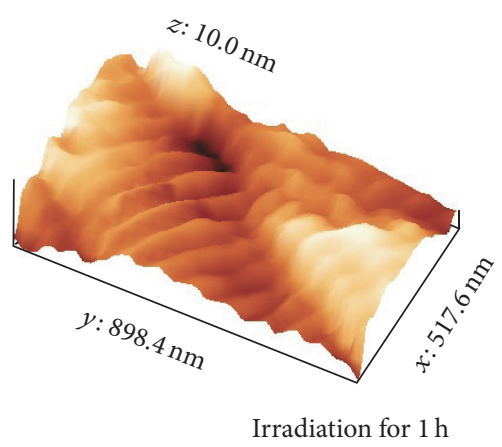

(b)

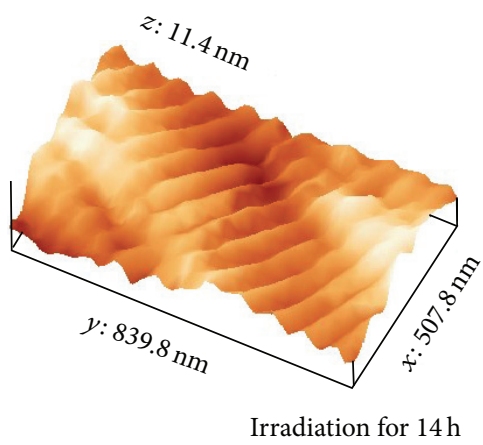

(c)

FIGURE 4: AFM topography images of UV-irradiated thin films. The figure demonstrates the same area on collagen thin film (original) after UV irradiation for 0 (a), 1 (b), and $14 \mathrm{~h}(\mathrm{c})$.

[118]. The combination of AFM with SHG techniques (imaging or signal measurements) can offer unique opportunities for investigating these collagen alterations under different pathological conditions.

In the literature we can see that the AFM-SHG combination has been applied in order to study collagen-rich tissues from tumor related sections [119-122]. The studies offered new information about the correlation with collagen organization with cancer cell invasion, the importance of collagen stiffening, and how the amount of tumor microenvironment components influence collagen characteristics in tumors. In this direction, in vitro experiments with collagen thin films can be used as control models of the ECM and/or tumor microenvironment. By using collagen thin films as models with predefined characteristic and studying the SHG signal intensity in correlation with collagen structure as it was provided with AFM imaging, it has been demonstrated that when the major polarization of the laser source and the main axis of the collagen fibers were parallel, the SHG signal was increased [116]. Also, it was confirmed that SHG emission was significantly lower from collagen fibers that accept thermally induced structural deformations compared to normal fibers $[116,117]$. The AFM-SHG combination can be used to unlock a number of unknown properties of collagen so as to develop new biomarkers for assessing collagen related diseases.

\section{AFM and Three-Dimensional Collagen Gels}

Although 2D collagen thin films have a variety of applications (e.g., covering a substrate so as to form a 2D model and culture cells on it), they lack the ability to mimic more precisely in vivo extracellular matrix conditions. Although 2D models, such as collagen thin films for culturing purposes, are used in the majority of in vitro studies [123], it is now generally accepted that $2 \mathrm{D}$ conditions cannot fully recapitulate the in vivo conditions as they present a number of limitations such as the lack of the three-dimensional (3D) physical cues, the lack of a way to take account of the high ECM stiffness, and the limited spatial distribution of adhesions to ECM [123]. In order to overcome some of these limitations, 3D in vitro models are increasingly receiving attention since they are more physiologically relevant.
For instance, 3D collagen gels have been developed to also take into consideration the mechanical forces that cells "feel" through interaction with ECM $[124,125]$. In that regard, there are several approaches reported in the literature [125]: (a) cells are cultured embedded in the gels [126-128], (b) cell spheroids are also embedded in collagen gels $[129,130]$, and (c) cells are cultured in a 3D "sandwich" collagen gel [131].

Despite the fact that 3D collagen models are very promising, their characterization is more complicated and tricky. Although AFM is a surface characterization technique, it can also be a significant tool for imaging and characterization of the structure and mechanical properties of these 3D gels (see Figure 5), which opens new avenues for the use of AFM in more physiologically relevant conditions. For instance, Tan et al. applied AFM imaging to investigate surface modifications that were induced by chitosan incorporation on collagen gels [132]. Also, AFM was used by Vicens-Zygmunt et al. to study the ribose-induced mechanical alterations in collagen gels by assessing their Young's elastic modulus [133] and by Nam et al. to explore strain stiffening of collagen gels [134]. Finally, in very recent publications, van Helvert and Friedl performed AFM nanoindentation in live-cell culture in order to directly address local elasticity changes generated by moving cells on fibrillar type I collagen gels [135].

\section{Advanced AFM Tools for Studying Collagen Collagen-Based Nanobiomaterials}

So far the unique capabilities of AFM topography characterization for collagen-based nanobiomaterials were presented. Although the nanocharacterization of collagen nanotopography may offer valuable information, AFM has many other advanced tools and every day new functions emerge. In this section some of the advanced and/or new features of AFM are presented.

Force Volume Mode. As it was presented in Section 2, in AFM force spectroscopy, the AFM can be used for measuring mechanical properties of the samples, such as the sample's Young modulus. An interesting extension of AFM force spectroscopy is the so-called "Force Volume Mode", which 


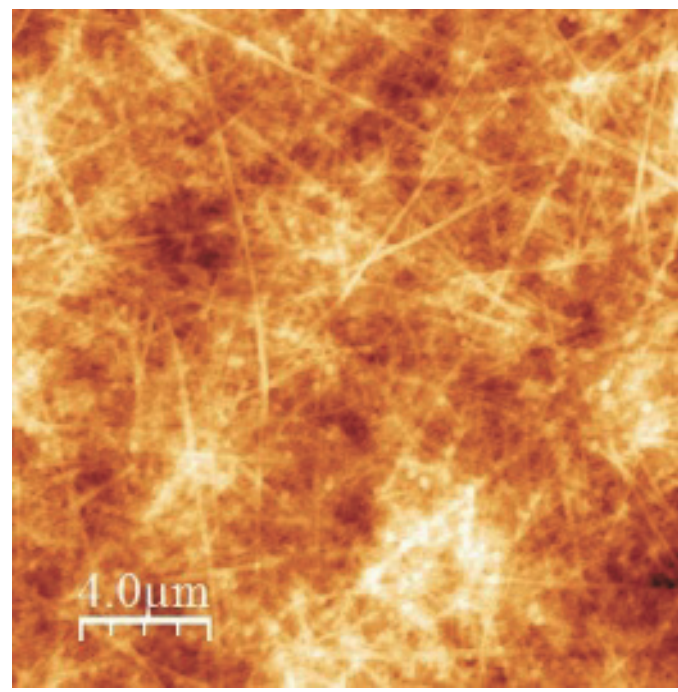

(a)

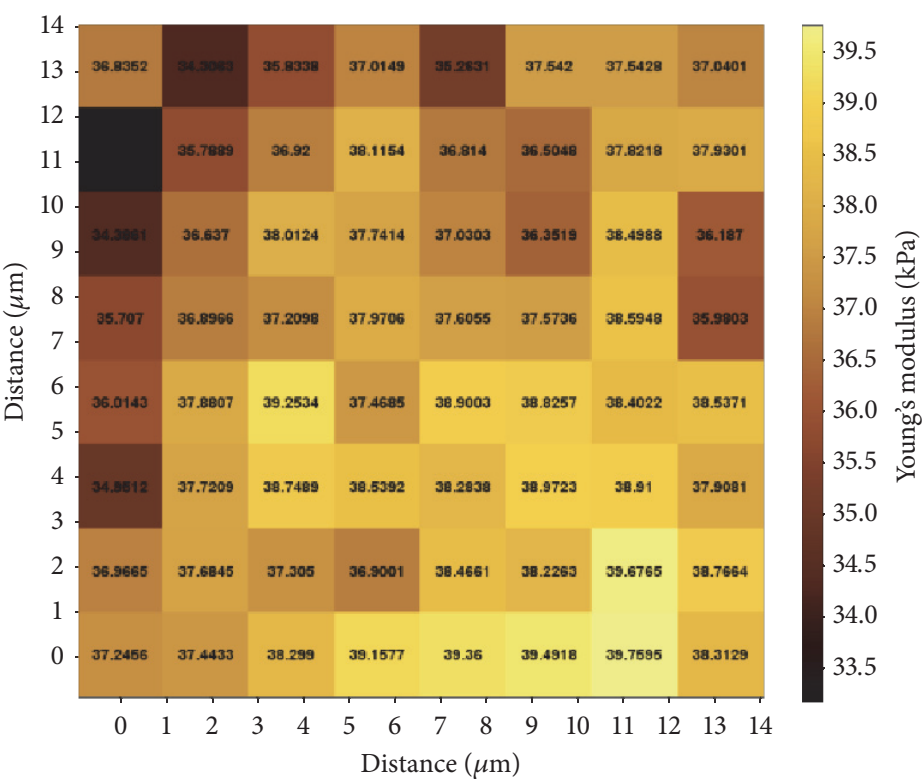

(b)

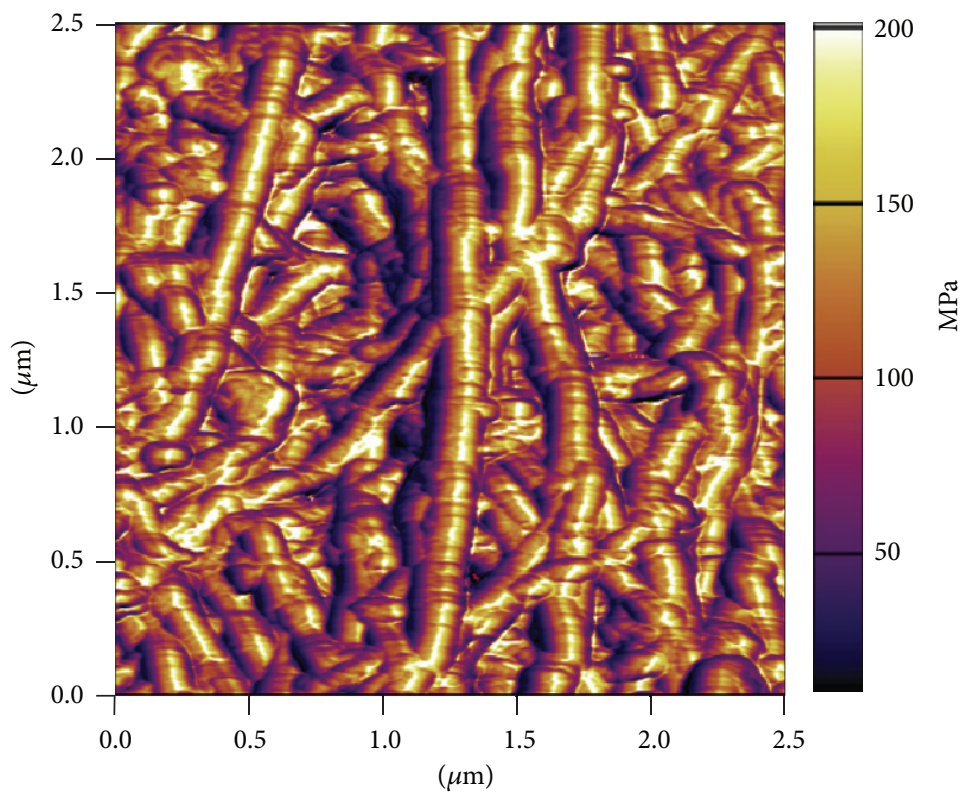

(c)

Figure 5: (a) AFM topography image of collagen gel (with Cypher ES, Asylum AFM system), (b) Force Volume map of collagen gel (with PicoPlus Molecular Imaging/Agilent AFM system and analysis with Atomic) software), and (c) AM-FM image of collagen gel (with Cypher ES, Asylum AFM system).

combines AFM imaging and force spectroscopy [43]. In this mode the sample's properties are measured in defined areas and not only in individual points. After acquiring forcedistance curves in all the areas of interest by nanoindenting each point separately, advanced calculations are used to calculate mechanical properties like stiffness (e.g., material Young's modulus). The final recorded data set in Volume Spectroscopy contains not only the three-dimensional image, but also force spectroscopy data for all the user-selected points in the image which can be presented as a Young modulus map of the region of interest (see Figure 5(b)).
AM-FM Viscoelastic Mapping. It is a very new AFM tool for nanomechanical characterization of materials, including biomaterials. AM-FM Mode has its roots in research on multifrequency and bimodal AFM, which is a dynamic forcebased method that can simultaneously map the topography and the nanomechanical properties of soft-matter surfaces $[136,137]$. AM-FM Mode provides mechanical information including Young's modulus and contact stiffness while operating at two cantilever resonances simultaneously [138]. Because AM-FM Mode works like tapping mode it has the advantages of this mode including fast scanning, high 
spatial resolution, and low forces applied to the sample (in contrast with the traditional force spectroscopy which is a time consuming procedure that is performed in contact mode). In Figure 5(c) an AM-FM Young modulus map of collagen gel is illustrated. As it can be seen the different Young moduli of collagen gel are clearly observed, while even the Dband periodicity in terms of stiffness can also be observed. This mode is very new and it is provided by specific AFM manufactures, but it has already demonstrated that it can be used for studying the elastic modulus of proteins [139] and small biological fibrils, like amyloid fibrils [140].

AFM and Combined Microscopy Techniques. AFM can be combined with other microscopes and imaging methods, such as optical microscopy and SEM. These combined modalities have unique capabilities and take advantage of all the properties of the involved modalities. Several research teams have already built sophisticated multifunctional microscopes [141], while a number of manufacturers have started producing of microscopes that combine AFM with other modalities. Coupling AFM with optical microscopy techniques (such as fluorescence microscopy and multiphoton microscopy) is very useful for the full characterization of cells and their microenvironment components, such as collagen matrix [142]. Optical microscopy can be used for imaging a large population of cells, a fact that offers capabilities for better statistical analysis. Furthermore, the use of fluorescent dyes can be used for labeling unique features of the cells [143]. As a result, the combined modality offers topographical characterization in the nanoscale simultaneously to a broader imaging field and biochemical capabilities. On the other hand, the AFM-SEM combination adds to the unique properties of AFM the advantages of SEM, which include the abilities to image rougher specimens with a larger depth of field and X-ray elemental analysis. The combination of the two microscopy modalities, either as separated systems or as hybrid combined modalities, can be very useful in life science research areas. For instance, the two modalities were used in order to investigate the behavior of myofibroblasts that were cultured on collagen-based substrates (e.g., collagen films and sponge), fully characterized by AFM and SEM [139]. In another study, hydrogels from synthetic collagen were used in order to study how transformed mammary epithelial cells interact with ECM with different characteristics (such as density and topography) [144].

\section{Conclusions}

This short review summarizes the use of AFM for novel collagen thin film formation and their applications for studying collagen thin film and 3D-gels formation and characteristics, collagen-substrate and cells interactions, and the collagenoptical radiation interactions (e.g., ultraviolet radiation, lowlevel laser irradiation, and second harmonic generation). AFM is a powerful multifunctional tool that can significantly contribute towards the development and the nanoscale characterization of novel nanomaterials.

\section{Competing Interests}

The author declares that there is no conflict of interests regarding the publication of this paper.

\section{Acknowledgments}

The research and the AFM images were taken by AS at the Biomedical Optics and Applied Biophysics Laboratory, National Technical University of Athens, Greece, and at the Cancer Biophysics Laboratory, University of Cyprus with a CPII (Bruker), a PicoPlus (Molecular Imaging/Agilent), and a Cypher ES (Asylum Research) AFM systems, respectively. This work was supported by the European Commission through a Horizon 2020, Marie SkłodowskaCurie Individual Fellowship (MSCA-IF-2014-658769-MYODESMOPLASIA).

\section{References}

[1] P. Fratzl, Collagen Structure and Mechanics, Springer, New York, NY, USA, 2008.

[2] G. T. Tihan, I. Rău, R. G. Zgârian, and M. V. Ghica, "Collagenbased biomaterials for ibuprofen delivery," Comptes Rendus Chimie, vol. 19, pp. 389-393, 2016.

[3] B. D. Walters and J. P. Stegemann, "Strategies for directing the structure and function of three-dimensional collagen biomaterials across length scales," Acta Biomaterialia, vol. 10, no. 4, pp. 1488-1501, 2014

[4] S. Ricard-Blum, "The collagen family," Cold Spring Harbor Perspectives in Biology, vol. 3, no. 1, pp. 1-19, 2011.

[5] D. J. S. Hulmes, "Collagen diversity, synthesis and assembly," Collagen: Structure and Mechanics, pp. 15-47, 2008.

[6] V. Hasirci, E. Vrana, P. Zorlutuna et al., "Nanobiomaterials: a review of the existing science and technology, and new approaches," Journal of Biomaterials Science, Polymer Edition, vol. 17, no. 11, pp. 1241-1268, 2006.

[7] P. Fratzl, "Collagen: structure and mechanics, an introduction," Collagen: Structure and Mechanics, pp. 1-13, 2008.

[8] A. Stylianou, D. Yova, and K. Politopoulos, "Atomic force microscopy quantitative and qualitative nanoscale characterization of collagen thin films," in Proceedings of the 5th International Conference on Emerging Technologies in Non-Destructive Testing (NDT '12), pp. 415-420, Ioannina, Greece, February 2012.

[9] S. Ricard-Blum, F. Ruggiero, and M. Van Der Rest, "The collagen superfamily," Topics in Current Chemistry, vol. 247, pp. 35-84, 2005.

[10] L. Bozec, G. van der Heijden, and M. Horton, "Collagen fibrils: nanoscale ropes," Biophysical Journal, vol. 92, no. 1, pp. 70-75, 2007.

[11] J. A. Petruska and A. J. Hodge, "A subunit model for the tropocollagen macromolecule," Proceedings of the National Academy of Sciences of the United States, vol. 51, pp. 871-876, 1964.

[12] J. M. Wallace, B. G. Orr, J. C. Marini, and M. M. B. Holl, "Nanoscale morphology of Type I collagen is altered in the Brtl mouse model of Osteogenesis Imperfecta," Journal of Structural Biology, vol. 173, no. 1, pp. 146-152, 2011. 
[13] C. A. Grant, M. A. Phillips, and N. H. Thomson, "Dynamic mechanical analysis of collagen fibrils at the nanoscale," Journal of the Mechanical Behavior of Biomedical Materials, vol. 5, no. 1, pp. 165-170, 2012.

[14] K. Poole, K. Khairy, J. Friedrichs et al., "Molecular-scale topographic cues induce the orientation and directional movement of fibroblasts on two-dimensional collagen surfaces," Journal of Molecular Biology, vol. 349, no. 2, pp. 380-386, 2005.

[15] D. R. Stamov, A. Müller, Y. Wegrowski, S. Brezillon, and C. M. Franz, "Quantitative analysis of type I collagen fibril regulation by lumican and decorin using AFM," Journal of Structural Biology, vol. 183, no. 3, pp. 394-403, 2013.

[16] V. P. Ivanova and A. I. Krivchenko, "A current viewpoint on structure and evolution of collagens. I. Fibrillar collagens," Journal of Evolutionary Biochemistry and Physiology, vol. 48, no. 2, pp. 127-139, 2012.

[17] M. D. Shoulders and R. T. Raines, "Collagen structure and stability, Annual Review of Biochemistry, vol. 78, pp. 929-958, 2009.

[18] M. K. Gordon and R. A. Hahn, "Collagens," Cell and Tissue Research, vol. 339, no. 1, pp. 247-257, 2010.

[19] L. Cen, W. Liu, L. Cui, W. Zhang, and Y. Cao, "Collagen tissue engineering: development of novel biomaterials and applications," Pediatric Research, vol. 63, no. 5, pp. 492-496, 2008.

[20] A. Stylianou and D. Yova, "Surface nanoscale imaging of collagen thin films by Atomic Force Microscopy," Materials Science and Engineering: C, vol. 33, no. 5, pp. 2947-2957, 2013.

[21] A. Stylianou, D. Yova, and K. Politopoulos, "Atomic force microscopy surface nanocharacterization of UV-irradiated collagen thin films," in Proceedings of the 12th IEEE International Conference on BioInformatics and BioEngineering (BIBE '12), Larnaca, Cyprus, November 2012.

[22] V. J. Morris, A. R. Kirby, and A. P. Gunning, Atomic Force Microscopy for Biologists, Imperial College Press, London, UK, 2008.

[23] D. P. Allison, N. P. Mortensen, C. J. Sullivan, and M. J. Doktycz, "Atomic force microscopy of biological samples," Wiley Interdisciplinary Reviews: Nanomedicine and Nanobiotechnology, vol. 2, no. 6, pp. 618-634, 2010.

[24] D. A. Cisneros, J. Friedrichs, A. Taubenberger, C. M. Franz, and D. J. Muller, "Creating ultrathin nanoscopic collagen matrices for biological and biotechnological applications," Small, vol. 3, no. 6, pp. 956-963, 2007.

[25] S. Zhuo, X. Zhu, J. Chen, and S. Xie, "Quantitative biomarkers of human skin photoaging based on intrinsic second harmonic generation signal," Scanning, vol. 35, no. 4, pp. 273-276, 2013.

[26] H. Yokota, J. Kaneshiro, and Y. Uesu, "Optical second harmonic generation microscopy as a tool of material diagnosis," Physics Research International, vol. 2012, Article ID 704634, 12 pages, 2012.

[27] P. Sowa, J. Rutkowska-Talipska, K. Rutkowski, B. KosztyłaHojna, and R. Rutkowski, "Optical radiation in modern medicine," Advances in Dermatology and Allergology, vol. 30, no. 4, pp. 246-251, 2013.

[28] M. H. Niemz, Laser-Tissue Interactions, Springer, Heidelberg, Germany, 2002.

[29] A. E. Tuer, S. Krouglov, N. Prent et al., "Nonlinear optical properties of type i collagen fibers studied by polarization dependent second harmonic generation microscopy," Journal of Physical Chemistry B, vol. 115, no. 44, pp. 12759-12769, 2011.
[30] R. M. Williams, W. R. Zipfel, and W. W. Webb, "Interpreting second-harmonic generation images of collagen I fibrils," Biophysical Journal, vol. 88, no. 2, pp. 1377-1386, 2005.

[31] A. Sionkowska, M. Wisniewski, J. Skopinska et al., “Thermal and mechanical properties of UV irradiated collagen/chitosan thin films," Polymer Degradation and Stability, vol. 91, no. 12, pp. 3026-3032, 2006.

[32] A. Torikai and H. Shibata, "Effect of ultraviolet radiation on photodegradation of collagen," Journal of Applied Polymer Science, vol. 73, no. 7, pp. 1259-1265, 1999.

[33] G. Binnig, C. F. Quate, and C. Gerber, "Atomic force microscope," Physical Review Letters, vol. 56, no. 9, pp. 930-933, 1986.

[34] N. Gadegaard, "Atomic force microscopy in biology: technology and techniques," Biotechnic and Histochemistry, vol. 81, no. 2-3, pp. 87-97, 2006.

[35] A. Stylianou, D. Yova, and E. Alexandratou, "Nanotopography of collagen thin films in correlation with fibroblast response," Journal of Nanophotonics, vol. 7, no. 1, Article ID 073590, 2013.

[36] A. Stylianou and T. Stylianopoulos, "Atomic force microscopy probing of cancer cells and tumor microenvironment components," BioNanoScience, vol. 6, no. 1, pp. 33-46, 2016.

[37] A. Stylianou, K. Politopoulos, and D. Yova, "Atomic force microscopy imaging of the nanoscale assembly of type i collagen on controlled polystyrene particles surfaces," in Proceedings of the 5th European Conference of the International Federation for Medical and Biological Engineering, pp. 1058-1061, September 2011.

[38] A. Stylianou, S. B. Kontomaris, M. Kyriazi, and D. Yova, "Surface characterization of collagen films by atomic force microscopy," in Proceedings of the 12th Mediterranean Conference on Medical and Biological Engineering and Computing (MEDICON '10), pp. 612-615, Chalkidiki, Greece, 2010.

[39] S. V. Kontomaris, A. Stylianou, D. Yova, and K. Politopoulos, "Mechanical properties of collagen fibrils on thin films by atomic force microscopy nanoindentation," in Proceedings of the 12th IEEE International Conference on BioInformatics and BioEngineering (BIBE '12), pp. 608-613, Larnaca, Cyprus, November 2012.

[40] A. Stylianou, S. V. Kontomaris, D. Yova, and G. Balogiannis, "AFM multimode imaging and nanoindetation method for assessing collagen nanoscale thin films heterogeneity," in Proceedings of the 13th Mediterranean Conference on Medical and Biological Engineering and Computing, vol. 41, 2014.

[41] S. V. Kontomaris, A. Stylianou, D. Yova, and G. Balogiannis, "The effects of UV irradiation on collagen D-band revealed by atomic force microscopy," Scanning, vol. 37, no. 2, pp. 101-111, 2015.

[42] Keysight-Technologies, Keysight 5500 Scanning Probe Microscope-User's Guide, N9410-90001, Keysight Technologies, 2014.

[43] W. Han and F. M. Serry, Force Spectroscopy with the Atomic Force Microscope-Application Note, Agilent Technologies, 2008.

[44] M. Stolz, R. Raiteri, A. U. Daniels, M. R. VanLandingham, W. Baschong, and U. Aebi, "Dynamic elastic modulus of porcine articular cartilage determined at two different levels of tissue organization by indentation-type atomic force microscopy," Biophysical Journal, vol. 86, no. 5, pp. 3269-3283, 2004.

[45] M. Stolz, R. Gottardi, R. Raiteri et al., "Early detection of aging cartilage and osteoarthritis in mice and patient samples using atomic force microscopy," Nature Nanotechnology, vol. 4, pp. 186-192, 2010. 
[46] W. C. Oliver and G. M. Pharr, "Measurement of hardness and elastic modulus by instrumented indentation: advances in understanding and refinements to methodology," Journal of Materials Research, vol. 19, no. 1, pp. 3-20, 2004.

[47] E. M. Darling, "Force scanning: a rapid, high-resolution approach for spatial mechanical property mapping," Nanotechnology, vol. 22, no. 17, 2011.

[48] C. Braunsmann, J. Seifert, J. Rheinlaender, and T. E. Schäffer, "High-speed force mapping on living cells with a small cantilever atomic force microscope," Review of Scientific Instruments, vol. 85, no. 7, Article ID 073703, 2014.

[49] A. Stylianou, S.-V. Kontomaris, and D. Yova, "Assessing collagen nanoscale thin films heterogeneity by AFM multi-mode imaging and nanoindetation for nanobiomedical applications," Micro and Nanosystems, vol. 6, no. 2, pp. 95-102, 2014.

[50] S. V. Kontomaris, D. Yova, A. Stylianou, and K. Politopoulos, "The significance of the percentage differences of young's modulus in the AFM nanoindentation procedure," Micro and Nanosystems, vol. 7, no. 2, pp. 86-97, 2015.

[51] H. Hertz, "Ueber die Berührung fester elastischer Körper," Journal für die Reine und Angewandte Mathematik, vol. 92, pp. 156-171, 1882.

[52] J. L. Mackay and S. Kumar, "Measuring the elastic properties of living cells with atomic force microscopy indentation," Methods in Molecular Biology, vol. 931, pp. 313-329, 2013.

[53] D. J. Müller and Y. F. Dufrêne, "Atomic force microscopy: a nanoscopic window on the cell surface," Trends in Cell Biology, vol. 21, no. 8, pp. 461-469, 2011.

[54] D. J. Müller and Y. F. Dufrêne, "Atomic force microscopy as a multifunctional molecular toolbox in nanobiotechnology," Nature Nanotechnology, vol. 3, no. 5, pp. 261-269, 2008.

[55] M. Li, L.-Q. Liu, N. Xi, and Y.-C. Wang, "Nanoscale monitoring of drug actions on cell membrane using atomic force microscopy," Acta Pharmacologica Sinica, vol. 36, no. 7, pp. 769782, 2015.

[56] X. Shi, X. Zhang, T. Xia, and X. Fang, "Living cell study at the single-molecule and single-cell levels by atomic force microscopy," Nanomedicine, vol. 7, no. 10, pp. 1625-1637, 2012.

[57] F. Variola, "Atomic force microscopy in biomaterials surface science," Physical Chemistry Chemical Physics, vol. 17, no. 5, pp. 2950-2959, 2015.

[58] B. Ohler, "Perspectives on over twenty years of life science research with atomic force microscopy and a look toward the future," Microscopy and Microanalysis, vol. 16, no. 2, pp. 10341035, 2010.

[59] D. Kirmizis and S. Logothetidis, "Atomic force microscopy probing in the measurement of cell mechanics," International Journal of Nanomedicine, vol. 5, no. 1, pp. 137-145, 2010.

[60] H. Q. Phong, S.-L. Wang, and M.-J. Wang, "Cell behaviors on micro-patterned porous thin films," Materials Science and Engineering B: Solid-State Materials for Advanced Technology, vol. 169, no. 1-3, pp. 94-100, 2010.

[61] C. Y. Tay, S. A. Irvine, F. Y. C. Boey, L. P. Tan, and S. Venkatraman, "Micro-/nano-engineered cellular responses for soft tissue engineering and biomedical applications," Small, vol. 7, no. 10, pp. 1361-1378, 2011.

[62] J. T. Elliott, A. Tona, J. T. Woodward, P. L. Jones, and A. L. Plant, "Thin films of collagen affect smooth muscle cell morphology," Langmuir, vol. 19, no. 5, pp. 1506-1514, 2003.
[63] F. H. Silver, J. W. Freeman, and G. P. Seehra, "Collagen selfassembly and the development of tendon mechanical properties," Journal of Biomechanics, vol. 36, no. 10, pp. 1529-1553, 2003.

[64] F. Jiang, K. Khairy, K. Poole, J. Howard, and D. J. Müller, "Creating nanoscopic collagen matrices using atomic force microscopy," Microscopy Research and Technique, vol. 64, no. 56, pp. 435-440, 2004.

[65] F. Jiang, H. Hörber, J. Howard, and D. J. Müller, "Assembly of collagen into microribbons: effects of $\mathrm{pH}$ and electrolytes," Journal of Structural Biology, vol. 148, no. 3, pp. 268-278, 2004.

[66] E. K. F. Yim, R. M. Reano, S. W. Pang, A. F. Yee, C. S. Chen, and K. W. Leong, "Nanopattern-induced changes in morphology and motility of smooth muscle cells," Biomaterials, vol. 26, no. 26, pp. 5405-5413, 2005.

[67] S. Zhong, W. E. Teo, X. Zhu, R. W. Beuerman, S. Ramakrishna, and L. Y. L. Yung, "An aligned nanofibrous collagen scaffold by electrospinning and its effects on in vitro fibroblast culture," Journal of Biomedical Materials Research Part A, vol. 79, no. 3, pp. 456-463, 2006.

[68] J. T. Elliott, J. T. Woodward, A. Umarji, Y. Mei, and A. Tona, "The effect of surface chemistry on the formation of thin films of native fibrillar collagen," Biomaterials, vol. 28 , no. 4, pp. 576585, 2007.

[69] C. C. Dupont-Gillain and P. G. Rouxhet, "AFM study of the interaction of collagen with polystyrene and plasma-oxidized polystyrene," Langmuir, vol. 17, no. 23, pp. 7261-7266, 2001.

[70] G. G. Biino and P. Gröning, "Cleavage mechanism and surface chemical characterization of phengitic muscovite and muscovite as constrained by X-ray Photoelectron Spectroscopy," Physics and Chemistry of Minerals, vol. 25, no. 2, pp. 168-181, 1998.

[71] F. Ostendorf, C. Schmitz, S. Hirth, A. Kühnle, J. J. Kolodziej, and M. Reichling, "How flat is an air-cleaved mica surface?" Nanotechnology, vol. 19, no. 30, 2008.

[72] H. Yang, S.-Y. Fung, M. Pritzker, and P. Chen, "Modification of hydrophilic and hydrophobic surfaced using an ioniccomplementary peptide," PLoS ONE, vol. 2, no. 12, Article ID e1325, 2007.

[73] S. Mulley, "Ordering and manipulation of MoS2 platelets on differently charged micas by atomic force microscopy," Journal of Materials Chemistry, vol. 6, no. 4, pp. 661-666, 1996.

[74] M. Sun, A. Stetco, and E. F. Merschrod, "Surface-templated formation of protein microfibril arrays," Langmuir, vol. 24, no. 10, pp. 5418-5421, 2008.

[75] J. K. Rainey and M. Cynthia Goh, "A statistically derived parameterization for the collagen triple-helix," Protein Science, vol. 11, no. 11, pp. 2748-2754, 2002.

[76] H.-W. Su, M.-S. Ho, and C.-M. Cheng, "Probing characteristics of collagen molecules on various surfaces via atomic force microscopy," Applied Physics Letters, vol. 100, no. 23, Article ID 233703, 2012.

[77] P. Lisboa, M.-B. Villiers, C. Brakha et al., "Fabrication of bio-functionalised polypyrrole nanoarrays for bio-molecular recognition," Micro and Nanosystems, vol. 3, no. 1, pp. 83-89, 2011.

[78] K. M. Brouwer, P. van Rensch, V. E. M. Harbers et al., "Evaluation of methods for the construction of collagenous scaffolds with a radial pore structure for tissue engineering," Journal of Tissue Engineering and Regenerative Medicine, vol. 5, no. 6, pp. 501-504, 2011. 
[79] A. L. Plant, K. Bhadriraju, T. A. Spurlin, and J. T. Elliott, "Cell response to matrix mechanics: focus on collagen," Biochimica et Biophysica Acta - Molecular Cell Research, vol. 1793, no. 5, pp. 893-902, 2009.

[80] X. F. Walboomers and J. A. Jansen, "Cell and tissue behavior on micro-grooved surfaces," Odontology, vol. 89, no. 1, pp. 2-11, 2001.

[81] W. A. Loesberg, J. te Riet, F. C. M. J. M. van Delft et al., "The threshold at which substrate nanogroove dimensions may influence fibroblast alignment and adhesion," Biomaterials, vol. 28, no. 27, pp. 3944-3951, 2007.

[82] S. Hou, X.-X. Li, X.-Y. Li et al., "Patterning of 293T fibroblasts on a mica surface," Analytical and Bioanalytical Chemistry, vol. 394, no. 8, pp. 2111-2117, 2009.

[83] S. Vanharanta and J. Massagué, "Field cancerization: something new under the sun," Cell, vol. 149, no. 6, pp. 1179-1181, 2012.

[84] T. T. Lu, Y. Z. Chen, T. Lu, and Y. Y. Zhang, "Mechanism of UV damage and the protective effect of natral products against UV damage," Chinese Pharmacological Bulletin, pp. 1655-1659, 2012.

[85] H. Ryssel, G. Germann, and E. Koellensperger, "An overview of current biomaterials in aesthetic soft tissue augmentation," European Journal of Plastic Surgery, vol. 35, no. 2, pp. 121-133, 2012.

[86] H. Suh and J.-C. Park, "Evaluation of calcification in porcine valves treated by ultraviolet ray and glutaraldehyde," Materials Science and Engineering C, vol. 13, no. 1-2, pp. 65-73, 2000.

[87] G. Fessel, J. Wernli, Y. Li, C. Gerber, and J. G. Snedeker, "Exogenous collagen cross-linking recovers tendon functional integrity in an experimental model of partial tear," Journal of Orthopaedic Research, vol. 30, no. 6, pp. 973-981, 2012.

[88] A. Gaspar, L. Moldovan, D. Constantin, A. M. Stanciuc, P. M. Sarbu Boeti, and I. C. Efrimescu, "Collagen-based scaffolds for skin tissue engineering," Journal of Medicine and Life, vol. 4, no. 2, pp. 172-177, 2011.

[89] N. N. Fathima, R. Suresh, J. R. Rao, B. U. Nair, and T. Ramasami, "Effect of UV irradiation on stabilized collagen: role of chromium(III)," Colloids and Surfaces B: Biointerfaces, vol. 62, no. 1, pp. 11-16, 2008.

[90] N. N. Fathima, R. Suresh, J. R. Rao, and B. U. Nair, "Effect of UV irradiation on the physicochemical properties of collagen stabilized using aldehydes," Journal of Applied Polymer Science, vol. 104, no. 6, pp. 3642-3648, 2007.

[91] K. Jariashvili, B. Madhan, B. Brodsky, A. Kuchava, L. Namicheishvili, and N. Metreveli, "Uv damage of collagen: insights from model collagen peptides," Biopolymers, vol. 97, no. 3, pp. 189-198, 2012.

[92] N. O. Metreveli, K. K. Jariashvili, L. O. Namicheishvili et al., "UV-vis and FT-IR spectra of ultraviolet irradiated collagen in the presence of antioxidant ascorbic acid," Ecotoxicology and Environmental Safety, vol. 73, no. 3, pp. 448-455, 2010.

[93] J. Skopinska-Wisniewska, A. Sionkowska, A. Kaminska, A. Kaznica, R. Jachimiak, and T. Drewa, "Surface characterization of collagen/elastin based biomaterials for tissue regeneration," Applied Surface Science, vol. 255, no. 19, pp. 8286-8292, 2009.

[94] A. Stylianou, D. Yova, E. Alexandratou, and A. Petri, "Atomic force imaging microscopy investigation of the interaction of ultraviolet radiation with collagen thin films," in Nanoscale Imaging, Sensing, and Actuation for Biomedical Applications X, vol. 8594 of Proceedings of SPIE, February 2013.

[95] A. Stylianou, D. Yova, and E. Alexandratou, "Investigation of the influence of UV irradiation on collagen thin films by AFM imaging," Materials Science and Engineering C, vol. 45, pp. 455468, 2014.

[96] C. Zink, H. Hall, D. M. Brunette, and N. D. Spencer, "Orthogonal nanometer-micrometer roughness gradients probe morphological influences on cell behavior," Biomaterials, vol. 33, no. 32, pp. 8055-8061, 2012.

[97] K. Anselme, A. Ponche, and M. Bigerelle, "Relative influence of surface topography and surface chemistry on cell response to bone implant materials. Part 2: biological aspects," Proceedings of the Institution of Mechanical Engineers, Part H: Journal of Engineering in Medicine, vol. 224, no. 12, pp. 1487-1507, 2010.

[98] U. Covani, L. Giacomelli, A. Krajewski et al., "Biomaterials for orthopedics: a roughness analysis by atomic force microscopy," Journal of Biomedical Materials Research-Part A, vol. 82, no. 3, pp. 723-730, 2007.

[99] A. Sionkowska, A. Płanecka, J. Kozłowska, and J. SkopińskaWiśniewska, "Surface properties of UV-irradiated poly(vinyl alcohol) films containing small amount of collagen," Applied Surface Science, vol. 255, no. 7, pp. 4135-4139, 2009.

[100] A. Sionkowska, J. Kozłowska, A. Płanecka, and J. SkopińskaWiśniewska, "Collagen fibrils in UV irradiated poly(vinyl pyrrolidone) films," Applied Surface Science, vol. 255, no. 5, pp. 2030-2039, 2008.

[101] Z. Cheng and S.-H. Teoh, "Surface modification of ultra thin poly ( $\varepsilon$-caprolactone) films using acrylic acid and collagen," Biomaterials, vol. 25, no. 11, pp. 1991-2001, 2004.

[102] M. Achilli, J. Lagueux, and D. Mantovani, "On the effects of UV$\mathrm{C}$ and $\mathrm{pH}$ on the mechanical behavior, molecular conformation and cell viability of collagen-based scaffold for vascular tissue engineering," Macromolecular Bioscience, vol. 10, no. 3, pp. 307316, 2010.

[103] A. B. Caruso and M. G. Dunn, "Changes in mechanical properties and cellularity during long-term culture of collagen fiber ACL reconstruction scaffolds," Journal of Biomedical Materials Research Part A, vol. 73, no. 4, pp. 388-397, 2005.

[104] N. Rajan, J. Lagueux, F. Couet, W. Pennock, D. Mantovani, and A. Sionkowska, "Low doses of ultraviolet radiation stimulate cell activity in collagen-based scaffolds," Biotechnology Progress, vol. 24, no. 4, pp. 884-889, 2008.

[105] S. J. Kew, J. H. Gwynne, D. Enea et al., "Regeneration and repair of tendon and ligament tissue using collagen fibre biomaterials," Acta Biomaterialia, vol. 7, no. 9, pp. 3237-3247, 2011.

[106] A. Sionkowska and T. Wess, "Mechanical properties of UV irradiated rat tail tendon (RTT) collagen," International Journal of Biological Macromolecules, vol. 34, no. 1-2, pp. 9-12, 2004.

[107] S. Anastase-Ravion, M. P. Carreno, C. Blondin et al., "Synergistic effects of glucose and ultraviolet irradiation on the physical properties of collagen," Journal of Biomedical Materials Research, vol. 60, no. 3, pp. 384-391, 2002.

[108] A. Stylianou and D. Yova, "Atomic force microscopy investigation of the interaction of low-level laser irradiation of collagen thin films in correlation with fibroblast response," Lasers in Medical Science, vol. 30, no. 9, pp. 2369-2379, 2015.

[109] R. W. Boyd, Nonlinear Optics, Academic Press, The Institute of Optics University of Rochester, New York, NY, USA, 1992.

[110] B.-M. Kim, J. Eichler, K. M. Reiser, A. M. Rubenchik, and L. B. Da Silva, "Collagen structure and nonlinear susceptibility: effects of heat, glycation, and enzymatic cleavage on second harmonic signal intensity," Lasers in Surgery and Medicine, vol. 27, no. 4, pp. 329-335, 2000. 
[111] T. Theodossiou, G. S. Rapti, V. Hovhannisyan, E. Georgiou, K. Politopoulos, and D. Yova, "Thermally induced irreversible conformational changes in collagen probed by optical second harmonic generation and laser-induced fluorescence," Lasers in Medical Science, vol. 17, no. 1, pp. 34-41, 2002.

[112] E. Brown, T. McKee, E. DiTomaso et al., "Dynamic imaging of collagen and its modulation in tumors in vivo using secondharmonic generation," Nature Medicine, vol. 9, no. 6, pp. 796800, 2003.

[113] S.-J. Lin, W. Lo, H.-Y. Tan et al., "Prediction of heat-induced Collagen shrinkage by use of second harmonic generation microscopy," Journal of Biomedical Optics, vol. 11, no. 3, Article ID 034020, 2006.

[114] P. J. Campagnola and L. M. Loew, "Second-harmonic imaging microscopy for visualizing biomolecular arrays in cells, tissues and organisms," Nature Biotechnology, vol. 21, no. 11, pp. 13561360, 2003.

[115] P. J. Campagnola and C.-Y. Dong, "Second harmonic generation microscopy: principles and applications to disease diagnosis," Laser and Photonics Reviews, vol. 5, no. 1, pp. 13-26, 2011.

[116] A. Stylianou, K. Politopoulos, M. Kyriazi, and D. Yova, "Combined information from AFM imaging and SHG signal analysis of collagen thin films," Biomedical Signal Processing and Control, vol. 6, no. 3, pp. 307-313, 2011.

[117] A. Stylianou, M. Kyriazi, K. Politopoulos, and D. Yova, "Combined SHG signal information with AFM imaging to assess conformational changes in collagen," in Proceedings of the 9th International Conference on Information Technology and Applications in Biomedicine (ITAB '09), Larnaka, Cyprus, November 2009.

[118] J. Myllyharju and K. I. Kivirikko, "Collagens and collagenrelated diseases," Annals of Medicine, vol. 33, no. 1, pp. 7-21, 2001.

[119] O. Maller, K. C. Hansen, T. R. Lyons et al., "Collagen architecture in pregnancy-induced protection from breast cancer," Journal of Cell Science, vol. 126, no. 18, pp. 4108-4120, 2013.

[120] J. G. Goetz, S. Minguet, I. Navarro-Lérida et al., "Biomechanical remodeling of the microenvironment by stromal caveolin-1 favors tumor invasion and metastasis," Cell, vol. 146, no. 1, pp. 148-163, 2011.

[121] M. W. Pickup, H. Laklai, I. Acerbi et al., "Stromally derived lysyl oxidase promotes metastasis of transforming growth factor- $\beta$ deficient mouse mammary carcinomas," Cancer Research, vol. 73, no. 17, pp. 5336-5346, 2013.

[122] R. Navab, D. Strumpf, C. To et al., "Integrin $\alpha 11 \beta 1$ regulates cancer stromal stiffness and promotes tumorigenicity and metastasis in non-small cell lung cancer," Oncogene, vol. 35, no. 15, pp. 1899-1908, 2016.

[123] M. Ferrarini, G. Mazzoleni, N. Steimberg, D. Belloni, and E. Ferrero, "Innovative models to assess multiple myeloma biology and the impact of drugs," in Multiple Myeloma-A Quick Reflection on the Fast Progress, R. Hajek, Ed., chapter 3, InTech, Rijeka, Croatia, 2013.

[124] B. Weigelt, C. M. Ghajar, and M. J. Bissell, “The need for complex 3D culture models to unravel novel pathways and identify accurate biomarkers in breast cancer," Advanced Drug Delivery Reviews, vol. 69-70, pp. 42-51, 2014.

[125] V. Gkretsi, A. Stylianou, P. Papageorgis, C. Polydorou, and T. Stylianopoulos, "Remodeling components of the tumor microenvironment to enhance cancer therapy," Frontiers in Oncology, vol. 5, article 214, 2015.
[126] S. Gehler, M. Baldassarre, Y. Lad et al., "Filamin A- $\beta 1$ integrin complex tunes epithelial cell response to matrix tension," Molecular Biology of the Cell, vol. 20, no. 14, pp. 3224-3238, 2009.

[127] C. S. Szot, C. F. Buchanan, J. W. Freeman, and M. N. Rylander, "3D in vitro bioengineered tumors based on collagen I hydrogels," Biomaterials, vol. 32, no. 31, pp. 7905-7912, 2011.

[128] B. N. Mason, A. Starchenko, R. M. Williams, L. J. Bonassar, and C. A. Reinhart-King, "Tuning three-dimensional collagen matrix stiffness independently of collagen concentration modulates endothelial cell behavior," Acta Biomaterialia, vol. 9, no. 1, pp. 4635-4644, 2013.

[129] L. J. Kaufman, C. P. Brangwynne, K. E. Kasza et al., "Glioma expansion in collagen I matrices: analyzing collagen concentration-dependent growth and motility patterns," Biophysical Journal, vol. 89, no. 1, pp. 635-650, 2005.

[130] M. Delarue, F. Montel, D. Vignjevic, J. Prost, J.-F. Joanny, and G. Cappello, "Compressive stress inhibits proliferation in tumor spheroids through a volume limitation," Biophysical Journal, vol. 107, no. 8, pp. 1821-1828, 2014.

[131] K. R. Levental, H. Yu, L. Kass et al., "Matrix crosslinking forces tumor progression by enhancing integrin signaling," Cell, vol. 139, no. 5, pp. 891-906, 2009.

[132] W. Tan, R. Krishnaraj, and T. A. Desai, "Influence of chitosan on cell viability and proliferation in three dimensional collage gels," in Proceedings of the 22nd Annual International Conference of the IEEE Engineering in Medicine and Biology Society, vol. 1502, pp. 1509-1524, Chicago, Ill, USA, July 2000.

[133] V. Vicens-Zygmunt, S. Estany, A. Colom et al., "Fibroblast viability and phenotypic changes within glycated stiffened three-dimensional collagen matrices," Respiratory Research, vol. 16, article 82, 2015.

[134] S. Nam, K. H. Hu, M. J. Butte, and O. Chaudhuri, "Strainenhanced stress relaxation impacts nonlinear elasticity in collagen gels," Proceedings of the National Academy of Sciences of the United States of America, vol. 113, no. 20, pp. 5492-5497, 2016.

[135] S. van Helvert and P. Friedl, "Strain stiffening of fibrillar collagen during individual and collective cell migration identified by AFM nanoindentation," ACS Applied Materials \& Interfaces, vol. 8, no. 34, pp. 21946-21955, 2016.

[136] R. Garcia and R. Proksch, "Nanomechanical mapping of soft matter by bimodal force microscopy," European Polymer Journal, vol. 49, no. 8, pp. 1897-1906, 2013.

[137] R. García and R. Pérez, "Dynamic atomic force microscopy methods," Surface Science Reports, vol. 47, no. 6-8, pp. 197-301, 2002.

[138] G. Chawla and S. D. Solares, "Mapping of conservative and dissipative interactions in bimodal atomic force microscopy using open-loop and phase-locked-loop control of the higher eigenmode," Applied Physics Letters, vol. 99, no. 7, Article ID 074103, 2011.

[139] R. A. B. Crabb, E. P. Chau, D. M. Decoteau, and A. Hubel, "Microstructural characteristics of extracellular matrix produced by stromal fibroblasts," Annals of Biomedical Engineering, vol. 34, no. 10, pp. 1615-1627, 2006.

[140] G. Lamour, C. K. Yip, H. Li, and J. Gsponer, "High intrinsic mechanical flexibility of mouse prion nanofibrils revealed by measurements of axial and radial young's moduli," ACS Nano, vol. 8, no. 4, pp. 3851-3861, 2014.

[141] R. Kassies, K. O. Van der Werf, A. Lenferink et al., "Combined AFM and confocal fluorescence microscope for applications in bio-nanotechnology," Journal of Microscopy, vol. 217, no. 1, pp. 109-116, 2005. 
[142] T. Ludwig, R. Kirmse, and K. Poole, "Challenges and approaches-probing tumor cell invasion by atomic force microscopy," in Modern Research and Educational Topics in Microscopy, A. Méndez-Vilas and J. Díaz, Eds., pp. 11-22, Formatex, 2007.

[143] I. Sokolov, "Chapter 1: atomic force microscopy in cancer cell research," in Cancer Nanotechnology, H. S. Nalwa and T. Webster, Eds., American Scientific, Valencia, Calif, USA, 2007.

[144] L. Cassereau, Y. A. Miroshnikova, G. Ou, J. Lakins, and V. M. Weaver, "A 3D tension bioreactor platform to study the interplay between ECM stiffness and tumor phenotype," Journal of Biotechnology, vol. 193, pp. 66-69, 2015. 

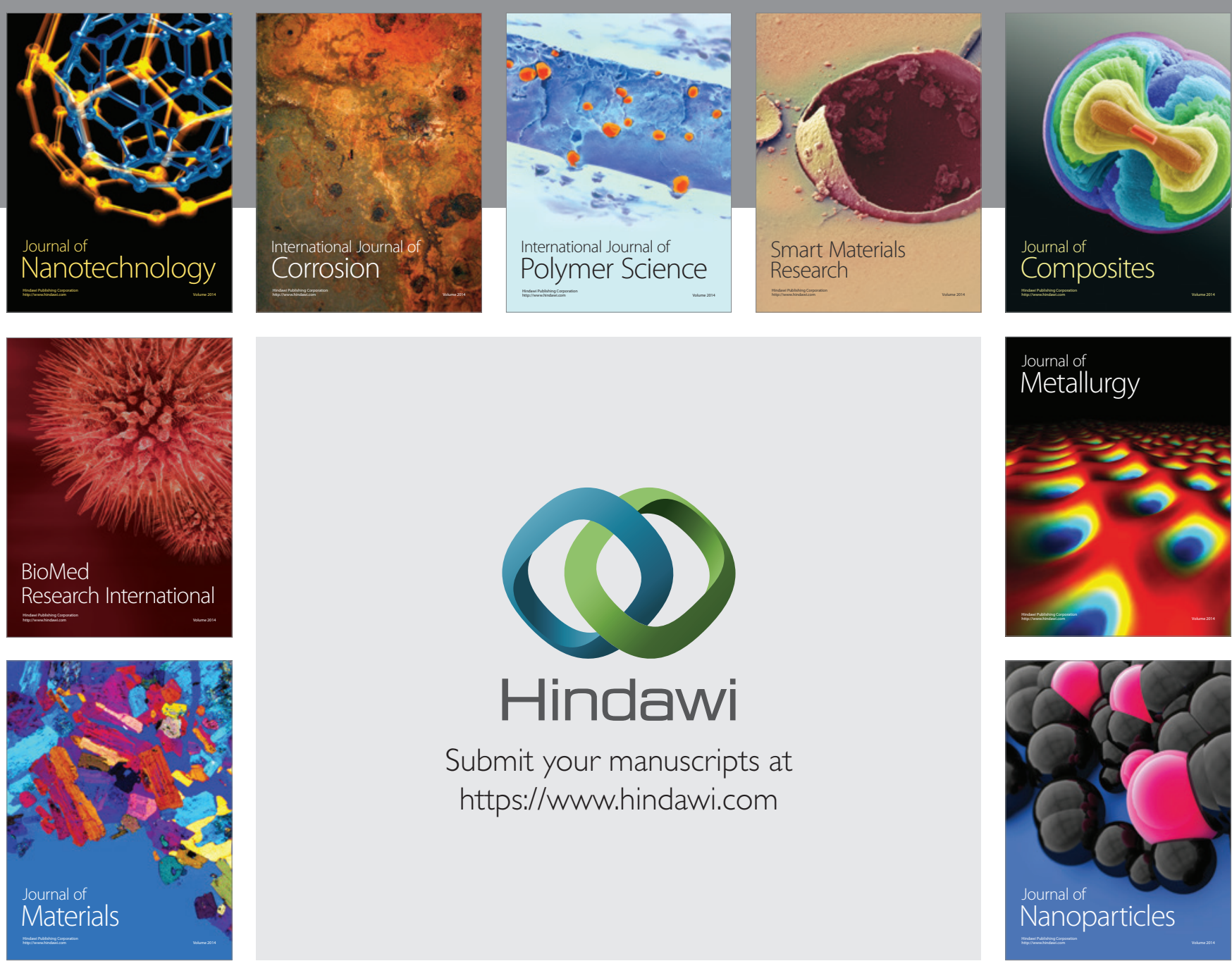

\section{Hindawi}

Submit your manuscripts at

https://www.hindawi.com

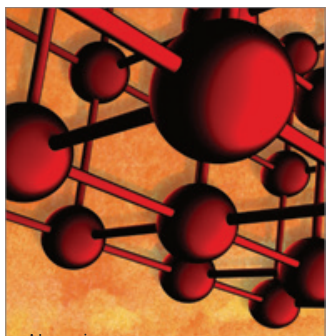

Materials Science and Engineering
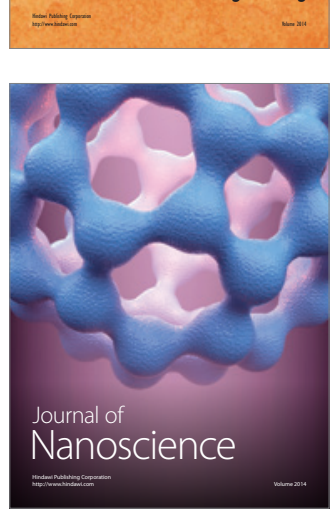
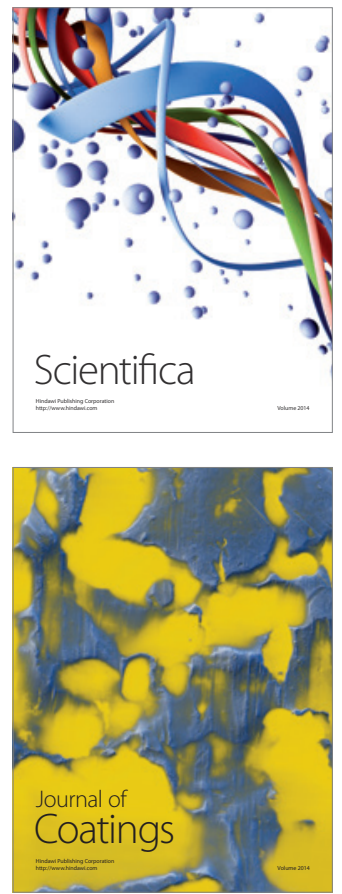
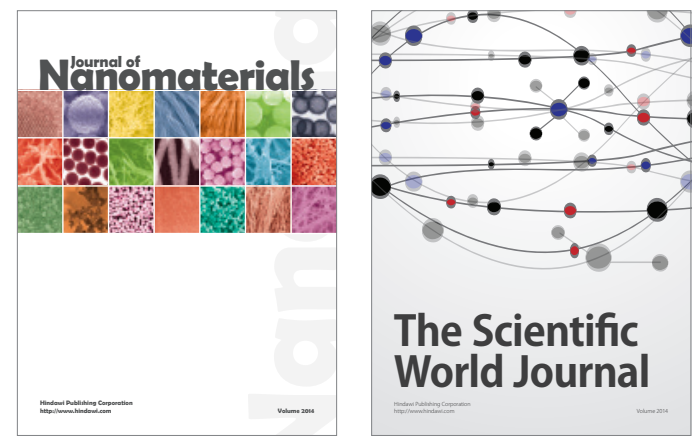

The Scientific World Journal
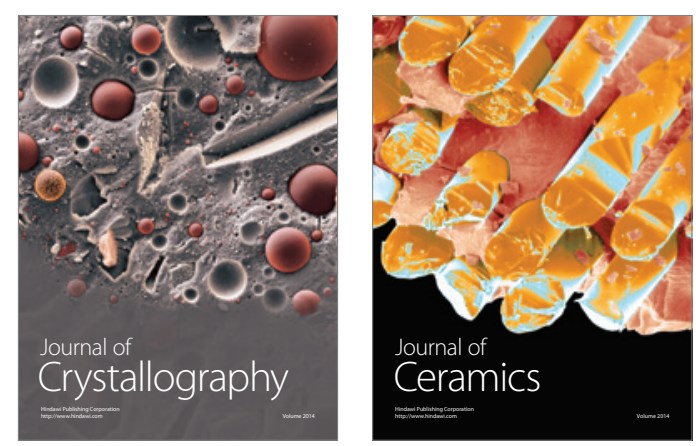
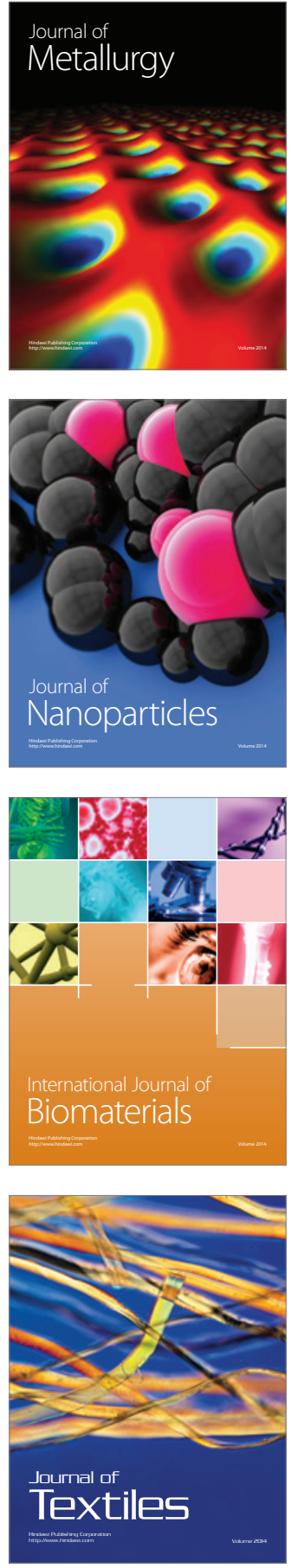